\title{
Proyecto educativo Olimpízate: objetivos, metodología y actividades para enseñar Olimpismo en los centros de educación secundaria Olimpízate, an educational project: objectives, methodology and activities to teach Olympism in secondary schools \\ José Luis Jorquera García, Alejandro Leiva Arcas, Juan Manuel Molina Morote, Antonio Sánchez Pato Universidad Católica de Murcia (España)
}

Resumen. Nuestra actual forma de practicar y entender el deporte, en cuanto a valores asociados se refiere, ha sido fijada principalmente por el Movimiento Olímpico. El Comité Olímpico Internacional reconoce tres valores fundamentales que definen el Olimpismo moderno: excelencia, amistad y respeto. Olimpízate es una iniciativa interdisciplinar, a la que se han adherido todos los departamentos didácticos de un centro de enseñanza secundaria, con el ánimo de incidir, desde diversas perspectivas, en la adquisición y proyección de los valores olímpicos. Los materiales se han presentado como una forma de promover el aprendizaje y la participación, estimular el pensamiento crítico, contribuir al desarrollo personal y social del alumno, descubrir la realidad olímpica, su historia y simbología y fomentar e inculcar los valores olímpicos en la comunidad educativa. Esta experiencia nos ha permitido explorar y poner en práctica las enormes posibilidades que tiene un centro educativo para incentivar la educación olímpica en los alumnos y alumnas de secundaria.

Palabras clave: Olimpismo, Valores Olímpicos, excelencia, amistad, respeto.

Abstract. Our current way to practice and understand the sport, in terms of associated values, has been set primarily by the Olympic Movement. The International Olympic Committee recognizes three fundamental values defining modern Olympism: excellence, friendship, and respect. Olimpízate is an interdisciplinary initiative that has joined all teaching departments of secondary schools, with the aim to foster the acquisition and projection of the Olympic Values from various perspectives. Materials have been presented as a way to promote learning and participation, to encourage critical thinking, to contribute to personal and social development of students, to discover the Olympic reality, its history and symbolism, and to promote and inculcate the Olympic values within the education community. This experience has allowed us to explore and implement the vast opportunities that schools have to encourage Olympic education in high school students.

Keywords: Olympism, Olympic Values, excellence, friendship, respect.

\section{Introducción}

El Olimpismo, tal y como lo entendemos hoy, es un concepto moderno creado por Pierre de Coubertin para definir una actitud, un conjunto de valores éticos; y que además, utiliza el deporte como vehículo para desarrollar su propósito pedagógico. Por lo tanto, es un término nuevo, que no tiene raíces o precursores en el Mundo Clásico. Sin embargo, como expresa Tavares (2006), el mundo académico no ha dado aún una definición suficientemente consensuada de Olimpismo.

Encuadrar teóricamente el Olimpismo pasa por organizar y sistematizar las ideas vertidas por el propio Coubertin en sus numerosos escritos, alocuciones, conferencias y correspondencias; especialmente en sus dos principales discursos ideológicos: El atletismo en el mundo moderno y los Juegos Olímpicos, en 1894 y Fundamentos filosóficos del Olimpismo moderno, de 1936 (Coubertin, 2012). Ciertamente, Coubertin no fue el primero que utilizó el deporte como medio pedagógico. De hecho, en su obra muestra una continua admiración por la educación inglesa, fundamentalmente por Thomas Arnold, quien utiliza el deporte con fines moralizantes en la Escuela de Rugby. Su intención era formar Christianes Gentlemen (Coubertin, 2012), esto es, alumnos educados en los valores de la perseverancia, la iniciativa, la autocrítica y el trabajo en equipo. Sin embargo, la gran aportación de Coubertin fue universalizar el carácter pedagógico del deporte en las Public Schools inglesas, dotarlo de símbolos (bandera y el fuego olímpico), rituales (entrada de los atletas, juramentos), así como de una base teórica con carácter educativo que busca fines más ambiciosos, como la paz mundial, la solidaridad o el desarrollo personal.

Lenk (1972; citado por DaCosta, 2002), uno de los más notables filósofos del deporte y de la idea olímpica, considera que una definición de Olimpismo requiere la inclusión del conjunto de valores básicos que identifican al Movimiento Olímpico. Vemos, por tanto, que la identificación de estos valores nos llevará ineludiblemente a la acotación conceptual del Olimpismo. Dichos valores se gestan a caballo entre los siglos XIX y XX, a la luz de una época conocida como modernidad, regida por teorías humanísticas y racionalistas que posibilitaron la pau-

Fecha recepción: 09-09-16. Fecha de aceptación: 27-03-17

José Luis Jorquera García

jorqueragarciacv@gmail.com latina secularización de la moral al margen de la religión, posibilitando a su vez la adopción de unos principios morales universales (Tavares, 2006).

Resumiendo, dos fenómenos sociales surgen a finales del siglo XIX y principios del XX: por un lado, el deporte, como manifestación lúdica y competitiva desarrollada en el contexto educativo anglosajón; y por otro, los valores asociados a la modernidad, con su fe inquebrantable en el progreso y la libertad humana. El gran mérito de Coubertin fue revestir al deporte de toda una carga ideológica de valores moralizantes, y que caracterizó desde entonces la manera que la sociedad tiene de practicar y entender el deporte. Además, utilizó éste como correa transmisora para objetivos educativos más amplios, estableciendo un movimiento internacional sin precedentes con la restauración de los Juegos Olímpicos. En la actualidad, el deporte es el escaparate donde más claramente se representan los valores imperantes en una sociedad y, por lo tanto, ofrece una oportunidad única para el reconocimiento y refuerzo de la identidad individual y colectiva (Weiss 2012).

\section{Los valores que definen el Olimpismo}

El propio Coubertin caracterizaba el Olimpismo no como un sistema, sino como una actitud espiritual, un estado de la mente, que aboga por la educación deportiva para todos y el espíritu caballeresco. Sin embargo, como apunta Lucas (1978), las ideas de Coubertin sobre la filosofía olímpica, a lo largo del tiempo y en sus numerosos escritos, «presentan algunas contradicciones, que en cambio, presentan siempre unos elementos identificativos y estables: la visión del Olimpismo como una filosofía pedagógica de religión, paz y belleza» (p. 78).

Solar (2003), nos remite a la definición de deporte del propio Coubertin, donde identifica cinco valores o nociones fundamentales: iniciativa, perseverancia, intensidad, búsqueda de la perfección y menosprecio por el peligro eventual. En la misma línea se posiciona Tavares (2006), para quien Coubertin y los autores que lo han interpretado, identificaron el Olimpismo con la combinación de una serie de valores románticos (el honor, la autosuperación, el juego limpio, el deber, la excelencia moral y el sentimiento de pertenencia) y los valores del iluminismo (universalismo, el valor de la competición, el individualismo y la fe en la educación).

Otros autores ofrecen interpretaciones más recientes acerca del Olimpismo. Según Chatziefstathiou y Henry (2012), fue una mezcla 
del filohelenismo, especialmente en cuanto a los antiguos valores de equilibrio entre cuerpo, mente y espíritu; y el deporte inglés. Por su parte, Weiss (2012) afirma que el Movimiento Olímpico ofrece como principal valor el juego limpio, es decir, la igualdad de oportunidades, el respeto al oponente y a las reglas.

Siguiendo a Maass (2007), Coubertin identificó para la conducción del Movimiento Olímpico los valores del respeto, el juego limpio, la búsqueda de la excelencia, la alegría en el esfuerzo y el equilibrio entre mente, cuerpo y voluntad. Del mismo modo se pronuncia Binder(2007), quien afirma que estos valores fundamentales son definidos a partir de los Principios Fundamentales del Olimpismo que recoge la Carta Olímpica. Para Maass, el Comité Olímpico Internacional (en adelante COI) debe fomentar y definir los valores olímpicos en el mundo contemporáneo. Por ello, el COI ha simplificado y aclarado recientemente los valores olímpicos, sin diluir el significado de los conceptos que promovió Coubertin hace más de cien años, en un esfuerzo por situarlos en el mundo contemporáneo y mostrar a la sociedad la misión del Movimiento Olímpico y así motivar las actividades y principios del COI (Maass, 2007)

Los valores olímpicos representan la base y el alma de todas las acciones del Movimiento Olímpico. Actualmente el COI reconoce tres valores fundamentales que definen el Olimpismo moderno: la excelencia, el respeto y la amistad (Maass, 2007):

Excelencia, que más allá de la mera victoria, significa ofrecer la mejor versión de uno mismo, superando las metas personales y comunes. Describe la calidad del esfuerzo que viene representada en el lema olímpico Citius, Altius, Fortius (más rápido, más alto, más fuerte).

Amistad, ya que utiliza el deporte como herramienta para el entendimiento. Su objetivo «es defender y fortalecer los vínculos entre las personas y los pueblos» (Maass, 2007, p.32), idea que viene representada por la llama olímpica, que con cada edición de los Juegos Olímpicos, es encendida en Olimpia y transportada hasta la ciudad anfitriona por miles de personas alrededor del mundo.

Respeto, que es sinónimo de juego limpio. Se orienta al respeto a uno mismo, a los demás competidores, al reglamento, a la modalidad deportiva en la que se participa y al medio ambiente. Es la condición que debe inspirar a todos los que participan en los programas del Movimiento Olímpico (Maass, 2007) y viene representado por la bandera olímpica.

Aunque dichos valores no se especifican en la Carta Olímpica, estos sí están implícitos en la formulación de los Principios Fundamentales del Olimpismo.

El Olimpismo se propone crear un estilo de vida basado en la alegría del esfuerzo, el valor educativo del buen ejemplo y el respeto por los principios éticos fundamentales universales.

El objetivo del Movimiento Olímpico es contribuir a construir un mundo mejor y más pacífico, educando a la juventud a través del deporte practicado sin discriminación de ningún tipo y dentro del espíritu olímpico, que exige comprensión mutua, espíritu de amistad, solidaridad y juego limpio (Carta Olímpica, 2015, p. 13).

Otros autores contemporáneos se muestran más escépticos a la hora de defender los valores moralizantes e idealistas que defiende el COI. Así, Maillard y Monnin (2014), explican que los sistemas educativos no pueden ignorar las enormes diferencias entre los valores que defiende el Movimiento Olímpico y la realidad de los Juegos Olímpicos, salpicados por continuos derroches económicos en infraestructuras, la excesiva comercialización de marcas publicitarias o la gran cobertura mediática dada casi en exclusividad a los deportistas vencedores. En el ámbito educativo, esta disparidad de perspectivas entre los postulados oficiales y la realidad que los alumnos y alumnas consumen diariamente en los medios de comunicación ha encontrado en la reciente metodología de la pedagogía crítica la manera más eficaz para hacer reflexionar a los estudiantes sobre episodios deportivos de actualidad y su posible desvinculación o no de los valores defendidos en la Carta Olímpica (Culpan \& Wigmore, 2010; Maillard \& Monnin, 2014).

Como hemos visto hasta ahora, el concepto de Olimpismo ha ido construyéndose paulativamente. Durante las últimas décadas numero- sos autores han ofrecido una clasificación de valores idealizados que deberían asociarse al deporte; clasificaciones, en algunos casos, demasiado extensas y ambiguas, en otros, muy ancladas en la terminología imperante en los textos coubertianos. Esta situación animó al COI a resumir estos valores en tres conceptos específicos (excelencia, amistad y respeto) y así facilitar el desarrollo de actividades encaminadas a la formación olímpica en programas educativos de diversa índole. La educación olímpica encuentra en la actualidad algunos escollos derivados del mercantilismo y excesivo elitismo en la alta competición, modelos negativos que distan mucho de los argumentos formativos, solidarios y pacifistas del Olimpismo. En los próximos apartados analizaremos cómo los más recientes programas educativos han desarrollado sus actividades, qué metodología e instrumentos han utilizado y de qué manera han repercutido en el enfoque y selección de tareas del Proyecto Educativo Olimpízate.

\section{La educación olímpica en el mundo y en España}

Fue a partir de los años setenta del siglo XX cuando surge el concepto de educación olímpica, mostrando los primeros proyectos, dirigidos a la educación primaria y secundaria, para celebración de los Juegos de Munich en 1972 y más tarde en Montreal 1976. En las últimas décadas, estas iniciativas se han ido consolidando y rebasando los intereses propios de los países anfitriones de Juegos Olímpicos. En la actualidad, los modelos y programas de educación olímpica existentes en el entorno educativo podemos clasificarlos, según Nikolaus (2013), de la siguiente forma: programas desarrollados por los países que albergan una edición olímpica, programas de ámbito internacional y programas nacionales para la educación en valores a través del deporte.

1. Programas de educación olímpica desarrollados por los países anfitriones de los Juegos Olímpicos. Desde Montreal 1976, con su primer modelo bilingüe de educación olímpica; hasta Río 2016, con la plataforma virtual de formación olímpica online Transforma, han sido muchas y variadas las iniciativas llevadas a cabo por los países y ciudades que han acogido unos Juegos Olímpicos. El mayor programa de educación olímpica desarrollado hasta ahora es el del Pekín 2008, en el que participaron 400 millones de alumnos y alumnas en más de 400.000 centros educativos (Naul, Binder, Rychtecky \& Culpan, 2017). Por su parte, el legado de Londres 2012, en cuanto a educación olímpica, sigue vigente con el proyecto International Inspiration, una organización benéfica que utiliza el deporte para la promoción de los derechos humanos, la igualdad de género y la inclusión de niños, niñas y jóvenes con diversidad funcional en actividades deportivas y sociales. Hasta el momento, esta iniciativa ha llegado a 25 millones de personas de Reino Unido, Jamaica, Gana, Zambia, Kenia, Etiopía, India y Bangladesh (Defroand, 2012).

2. Programas de educación olímpica internacionales, centrados en los valores a través del deporte y extrapolables a las diferentes áreas culturales de todo el mundo. Podemos destacar, entre todos, el Olympic Values Education Programme (OVEP), desarrollado por el COI o la Coubertin Academy. Veamos a continuación resumidamente algunas de las estrategias desarrolladas por el COI para promover los valores olímpicos y la educación olímpica entre los jóvenes:

La más ambiciosa de estas iniciativas surge en la 119 Sesión del CIO, el 5 de julio de 2007, en la ciudad de Guatemala. Se trata de la celebración de los Youth Olympic Games (YOG.), con atletas de todo el mundo con edades comprendidas entre los 15 y 18 años. La primera edición de verano tuvo lugar en Singapur en el año 2010, mientras que la primera edición de los deportes de invierno se celebró en Innsbruck, en 2012. Una de las características de estos Juegos es que atletas, espectadores y periodistas forman parte del Programa Educativo y Cultural, basado en cinco temas principales: Olimpismo, Responsabilidad social, Habilidades, Expresión y Bienestar y Estilo de vida saludable (Makris \& Georgiadis, 2013).

- Para Naul (2008) una de las principales estrategias del COI para promover los valores olímpicos es el anteriormente mencionado OVEP. Se trata de una herramienta que tiene como objetivo mantener el interés de los jóvenes por el deporte y promover el Olimpismo. Para 
ello, se basa en un manual (disponible en francés e inglés) de 136 páginas y que además incluye un conjunto de iniciativas educativas dirigidas a alumnos de entre ocho y dieciocho años.

Como podemos observar, estas dos estrategias denotan una especial preocupación del Movimiento Olímpico por los jóvenes y por fomentar un entramado conjunto de valores humanísticos, que va más allá del escaparate mediático que supone la celebración de unos Juegos Olímpicos. La labor pedagógica comprende la inclusión de la filosofía olímpica en los centros educativos, con la publicación de materiales didácticos para diferentes edades; y la utilización del deporte y la cultura como transmisores de valores y prácticas que permitan la construcción de un mundo más pacífico, tolerante y respetuoso con el medio ambiente. Hacemos nuestras las palabras de Solar (2003) cuando afirma que «los valores que Coubertin añade al deporte son en realidad sus exigencias morales a la juventud, y la juventud la depositaria de todo el objetivo de los Juegos Olímpicos» (p. 142).

3. Programas de educación olímpica nacionales, apoyados por los Comités y las Academias Olímpicas Nacionales, como los desarrollados en Australia, Nueva Zelanda, Francia o Alemania (Naul et al., 2017).

Dentro de este bloque, destacamos los más recientes programas desarrollados en España que han tratado los ideales olímpicos y que han sido impulsados desde organismos gubernamentales, universidades y centros educativos. Entre todos ellos destacamos especialmente tres:

«Ciudadanía Olímpica», de 2008, desarrollado por la Universidad Camilo José Cela, el Comité Olímpico Español y el Ministerio de Educación Cultura y Deporte. A través de una página web, docentes y estudiantes pueden acceder a un conjunto de herramientas descargables y en línea para trabajar sobre Olimpismo. Todas las actividades (recortes de prensa, vídeos, entrevistas o imágenes) se organizan en torno a tres valores identificados como esfuerzo-compromiso, integración-participación y respeto-compañerismo.

«Olimpismo en la Escuela», de 2011, que, desarrollado por la FundaciónAndalucía Olímpica, facilita una unidad didáctica de cinco sesiones, dirigida a alumnos y alumnas de educación primaria. Este programa está concebido para desarrollarse en las clases de educación física, dentro de su horario lectivo; aunque también es posible llevarlo a cabo durante una semana cultural del centro. Consta de una parte teórica en la que dos personajes de ficción (Diágoras y Teresa) narran a través de 17 comics famosas anécdotas de esfuerzo, compañerismo y superación personal protagonizadas por estrellas del deporte antiguas y de actualidad. Además. «Olimpismo en la Escuela» contiene una parte práctica, en la que se propicia que los estudiantes puedan conocer la simbología olímpica y desarrollar habilidades motrices en distintas competiciones deportivas (Arévalo \& Sotoca, 2014).

- «Héroes Olímpicos» y «Todos Olímpicos», de 2007; dos iniciativas dirigidas a educación primaria y secundaria respectivamente y que ha sido desarrollada por el Comité Olímpico Español y el Consejo Superior de Deportes. Su objetivo es la promoción de los valores de igualdad, respeto, constancia y esfuerzo, acercando el deporte de alto rendimiento a los alumnos y alumnas. Consiste en conferencias sobre los valores olímpicos a cargo de deportistas como Carolina Pascual, Rafa Lozano, Montse Puche o Joan Lino, entre otros, seguidos por una clase práctica en la que los escolares practican modalidades deportivas específicas bajo su dirección. En todas sus ediciones, esta campaña ha podido llegar hasta casi 100.000 niños y jóvenes de $3^{\circ}$ de primaria y $1^{\circ}$ deEducación Secundaria Obligatoria(ESO).

En la mayor parte de estos modelos, la atención se centra en aspectos informativos y estructurales acerca del Movimiento Olímpico y la celebración de los Juegos cuatrienales, exponiendo a los deportistas olímpicos como ejemplos para los jóvenes, no solo por sus logros deportivos, sino también por su actitud dentro y fuera del terreno de juego. Igualmente, son destacables las conocidas «Olimpiadas escolares», en las que priman las competiciones multideportivas entre los alumnos y alumnas de un mismo centro educativo o de varios. Sin embargo, en los últimos años, surgen voces discordantes que abogan por un cambio sustancial en la metodología seguida en los programas de educación olímpica. Para Hernández (2014, p. 134) «es preciso superar los discursos extremadamente idealizados basados en experiencias personales, héroes u orígenes culturales del deporte». En la misma línea se posicionan Monjas, Ponce y Gea (2015), cuando afirman que «el deporte puede ser un recurso educativo de primer orden, pero también puede generar aprendizajes no deseados: conductas discriminatorias y ejemplos negativos relacionados con la desigualdad» (p. 276).

Así, centrándonos en la realidad educativa española, destacamos algunos de los más recientes estudios acerca de la percepción que los jóvenes tienen sobre los valores asociados a la práctica deportiva. Calle y Martínez (2015) afirman que «existen dos valores propuestos en el ideario olímpico que vienen siendo desarrollados en los centros docentes españoles estudiados: el juego limpio y el respeto a los demás» (p. 357). Un trabajo de investigación llevado a cabo en Galicia (Martínez, et al., 2016) concluye que los valores más importantes para los alumnos y alumnas de Educación Secundaria Obligatoria y Bachillerato son la diversión, el esfuerzo, el juego limpio, hacer amigos y la deportividad. En la misma línea, Meroño, Bada, Sánchez-Pato, Calderón y FiguerMontero (2016), tras aplicar a un grupo de escolares de secundaria un programa basado en el Movimiento Olímpico para educar en valores, afirman que el valor educativo más puntuado fue el juego limpio en ambos géneros. Entre los chicos se dio un alto valor al respeto, el esfuerzo y la excelencia. Por su parte, las chicas destacaron la superación, la amistad y la igualdad.

Por otro lado, debemos considerar que existen multitud de agentes sociales que ejercen su influencia en la adquisición de valores: familia, medios de comunicación (marketing ligado a necesidades de consumo), grupos de amigos o el entorno cultural. Los jóvenes deportistas tienen como principal referente a los entrenadores. Sin embargo, un novedoso estudio desarrollado en Portugal (Rocha, Martínez, Mateos \& PenaPérez, 2016) revela la escasa formación de los entrenadores deportivos sobre temática olímpica y sus ideales. La investigación expone que los entrenadores contemplan, como valores asociados a los Juegos Olímpicos, la excelencia, seguido del éxito y el resultado. Estas conclusiones nos hacen plantearnos que sería conveniente extender los programas de educación olímpica no solo a los escolares, sino también a las instituciones encargadas de la formación de profesionales del deporte y la educación física.

El proyecto educativo Olimpízate va más allá y se presenta como una iniciativa educativa de carácter interdisciplinar que aborda la educación olímpica incidiendo especialmente en los valores. Pero estos no pueden ser enseñados ni aprendidos de la misma forma que los conceptos. Como afirman Martínez, Pena-Pérez y Mateos (2016): «para educar en valores no podemos hacer uso de procesos memorísticos o incluso de la simple presentación teórica de las bondades (o maldades) de acciones o actitudes. Debemos conseguir que los individuos asimilen conceptos» (p. 227). Proyectos similares, sobre Olimpismo y de carácter interdisciplinar, hemos visto en los últimos años: en primaria, Peixoto (2014) plantea un programa que involucra a todas las materias curriculares y que se extiende durante todo un curso escolar, incorporando igualmente actividades complementarias con tintes olímpicos, como el día de la paz o un carnaval olímpico. Interesante también es la propuesta de Lioumpi, Georgiadis y Mountakis (2014), centrada en el fomento de actitudes positivas relacionadas con la ética del deporte y los valores en las escuelas griegas. En educación secundaria destacamos el modelo de intervención presentado por Herrero (2015), que plantea los valores olímpicos en cinco ámbitos y luego los relaciona con todos los elementos del currículo, a fin de organizar las distintas actividades de enseñanza y aprendizaje. Desde luego, las futuras iniciativas para fomentar la educación olímpica, como indica Binder (2010), deben entender «la emoción y la imaginación como herramientas pedagógicas» (p.17). El proyecto de educación olímpica Olimpízate aglutina muchas de las iniciativas planteadas hasta ahora, incluso aquellas que no han tratado de forma específica el Olimpismo, sino que se han centrado en los valores éticos universales que pueden ser fomentados a través del deporte (Prat, Soler, Ventura \& Tirado, 2011; Martínez, 2011). 


\section{El proyecto Olimpízate. Contextualización}

Durante los últimos años, el Centro de Estudios Olímpicos de la Universidad Católica de Murcia (UCAM-CEO) ha venido desarrollando diversos materiales didácticos específicos sobre Olimpismo. Se trata de un conjunto de contenidos y experiencias realizadas por y para docentes a desarrollar en el aula, abarcando las etapas de primaria, secundaria y estudios universitarios. Los materiales se presentan como una forma de promover el aprendizaje y la participación, estimular el pensamiento crítico, contribuir al desarrollo personal y social del alumno, descubrir la realidad olímpica, su historia y simbología y fomentare inculcar los valores olímpicos.

Olimpízate es un proyecto que hemos desarrollado de forma pionera en el Instituto público de Educación Secundaria (IES) Beneche de la localidad de Nerpio, en la provincia de Albacete, en plena Sierra del Segura. El municipio cuenta con aproximadamente 1.500 habitantes, dedicados en su amplia mayoría al sector primario y al turismo rural. Por su parte, en el centro estudian un total de 53 estudiantes entre $1^{\circ}$ y $4^{\circ}$ de ESO y cuenta con una plantilla de 13 profesores. Esta propuesta educativa de carácter interdisciplinar ha sido desarrollada con alumnos y alumnas de $4^{\circ}$ de ESO (15-17 años). Además, han colaborado todos los departamentos didácticos, con el ánimo de incidir, desde diversas perspectivas, en la adquisición y proyección de los valores olímpicos. De esta forma, han participado un total de 25 alumnos y alumnas y 13 profesores de secundaria. Todos los docentes han intervenido en la experiencia a través de sus asignaturas, bajo la coordinación del profesor de educación física. Si bien es cierto, las horas dedicadas a Olimpízate han sido homogéneas en todas las materias (dos horas lectivas semanales por área), no dando protagonismo a unas sobre otras. La planificación, control y evaluación de esta iniciativa contó con un claustro de profesores extraordinario dos meses antes de su puesta en práctica, una reunión semanal, y finalmente, una sesión de evaluación.

En un primer momento, procedemos a la concreción de las llamadas dimensiones pedagógicas que abarca este proyecto. Esta clasificación nos permitirá organizar las actividades específicas por Departamentos Curriculares, así como la relación con los elementos de Currículo, y por tanto, la justificación de Olimpízate dentro de la Programación Didáctica. Nos hemos basado en las directrices que establece la OVEP, desde el Comité Olímpico Internacional, que identifica como valores educativos en el Olimpismo los siguientes: joy of effort, fair play, respect, pursuit of excellence, balance between body, will and mind. Además, incide en el conocimiento del pasado y presente de los Juegos Olímpicos (Binder, 2007). De esta forma, planteamos la siguiente secuenciación:

- Conocimiento olímpico

- Excelencia

\section{- Amistad}

- Respeto

A los tres valores fundamentales que definen el Olimpismo le hemos añadido la dimensión de «Conocimiento olímpico», con la que pretendemos indagar en los hitos más sobresalientes de la historia de los Juegos Olímpicos modernos, así como en sus aspectos estructurales y simbología (Shcherbashyn, 2014). No obstante, esta dimensión quedará relegada a un segundo plano durante este proyecto, puesto que los alumnos y alumnas ya habían trabajado estos contenidos en cursos anteriores dentro de la asignatura de educación física.

En primer lugar, debemos destacar que en la actual Ley Orgánica para la Mejora de la Calidad Educativa (LOMCE, 2013) aparece la incorporación de las denominadas competencias básicas, siguiendo las directrices del espacio educativo europeo. Estas competencias suponen un instrumento para que el alumno adquiera los conocimientos necesarios para resolver los problemas que depara la sociedad de forma autónoma y creativa.

Veamos a continuación, de forma resumida en la Tabla 1, la relación existente entre las dimensiones pedagógicas de Olimpízate y las competencias básicas curriculares, según el Real Decreto 1105/2014, de 26 de diciembre, por el que se establece el currículo básico de la Educación Secundaria Obligatoria y del Bachillerato.

\begin{tabular}{|c|c|}
\hline Competencias básicas & Dimensiones pedagógicas \\
\hline a. Comunicación lingüística & -Conocimiento olímpico \\
\hline $\begin{array}{l}\text { b. Competencia matemática y competencias básicas en } \\
\text { ciencia y tecnología }\end{array}$ & $\begin{array}{l}\text {-Conocimiento olímpico } \\
\text { - Excelencia }\end{array}$ \\
\hline c. Competencia digital & $\begin{array}{l}\text { - Conocimiento olímpico } \\
\text { - Amistad }\end{array}$ \\
\hline d. Aprender a aprender & $\begin{array}{l}\text { - Conocimiento olímpico } \\
\text { - Excelencia } \\
\text { - Respeto } \\
\text { - Amistad }\end{array}$ \\
\hline e. Competencias sociales y cívicas & $\begin{array}{l}\text { - Amistad } \\
\text { - Respeto }\end{array}$ \\
\hline f. Sentido de iniciativa y espíritu emprendedor & $\begin{array}{l}\text { - Excelencia } \\
\text { - Respeto } \\
\text { - Amistad } \\
\end{array}$ \\
\hline g. Conciencia y expresiones culturales & $\begin{array}{l}\text { - Conocimiento olímpico } \\
\text { - Excelencia } \\
\text { - Respeto }\end{array}$ \\
\hline
\end{tabular}

Igualmente, podemos establecer una relación entre los objetivos generales de la etapa y las dimensiones pedagógicas de Olimpízate, según el Real Decreto 1105/2014, de 26 de diciembre, por el que se establece el currículo básico de la Educación Secundaria Obligatoria y del Bachillerato (Tabla 2).

Observamos cómo Olimpízate da respuesta al tratamiento interdisciplinar que pretendemos. Este modelo clasifica y relaciona los valores olímpicos con los elementos curriculares de la etapa de la ESO,

Tabla 2 .

Objetivos Generales de la Etapa de Educación Secundaria Obligatoria y dimensiones pedagógicas Objetivensiones pedagógicas

\begin{tabular}{|c|c|}
\hline $\begin{array}{l}\text { a) Asumir responsablemente sus deberes, conocer y ejercer sus derechos en el respeto a los demás, practicar la tolerancia, la cooperación y la solidaridad entre las personas } \\
\text { y grupos, ejercitarse en el diálogo afianzando los derechos humanos y la igualdad de trato y de oportunidades entre mujeres y hombres, como valores comunes de una } \\
\text { sociedad plural y prepararse para el ejercicio de la ciudadanía democrática. }\end{array}$ & $\begin{array}{l}\text { - Amistad } \\
\text { - Respeto }\end{array}$ \\
\hline $\begin{array}{l}\text { b) Desarrollar y consolidar hábitos de disciplina, estudio y trabajo individual y en equipo como condición necesaria para una realización eficaz de las tareas del aprendizaje } \\
\text { y como medio de desarrollo personal. }\end{array}$ & $\begin{array}{l}\text { - Excelencia } \\
\text { - Respeto }\end{array}$ \\
\hline $\begin{array}{l}\text { c) Valorar y respetar la diferencia de sexos y la igualdad de derechos y oportunidades entre ellos. Rechazar la discriminación de las personas por razón de sexo o por } \\
\text { cualquier otra condición o circunstancia personal o social. Rechazar los estereotipos que supongan discriminación entre hombres y mujeres, así como cualquier } \\
\text { manifestación de violencia contra la mujer. }\end{array}$ & \\
\hline $\begin{array}{l}\text { d) Fortalecer sus capacidades afectivas en todos los ámbitos de la personalidad y en sus relaciones con los demás, así como rechazar la violencia, los prejuicios de } \\
\text { cualquier tipo, los comportamientos sexistas y resolver pacíficamente los conflictos. }\end{array}$ & $\begin{array}{l}\text {-Respeto } \\
\text { - Amistad }\end{array}$ \\
\hline $\begin{array}{l}\text { e) Desarrollar destrezas básicas en la utilización de las fuentes de información para, con sentido crítico, adquirir nuevos conocimientos. Adquirir una preparación básica en } \\
\text { el campo de las tecnologías, especialmente las de la información y la comunicación. }\end{array}$ & $\begin{array}{l}\text { - Conocimiento olímpico } \\
\text { - Excelencia }\end{array}$ \\
\hline $\begin{array}{l}\text { f) Concebir el conocimiento científico como un saber integrado, que se estructura en distintas disciplinas, así como conocer y aplicar los métodos para identificar los } \\
\text { problemas en los diversos campos del conocimiento y de la experiencia. }\end{array}$ & $\begin{array}{l}\text { - Excelencia } \\
\text { - Conocimiento olímpico }\end{array}$ \\
\hline $\begin{array}{l}\text { g) Desarrollar el espíritu emprendedor y la confianza en sí mismo, la participación, el sentido crítico, la iniciativa personal y la capacidad para aprender a aprender, } \\
\text { planificar, tomar decisiones y asumir responsabilidades. }\end{array}$ & $\begin{array}{l}\text { - Excelencia } \\
\text { - Respeto }\end{array}$ \\
\hline $\begin{array}{l}\text { h) Comprender y expresar con corrección, oralmente y por escrito, en la lengua castellana y, si la hubiere, en la lengua cooficial de la Comunidad Autónoma, textos y } \\
\text { mensajes complejos, e iniciarse en el conocimiento, la lectura y el estudio de la literatura. }\end{array}$ & $\begin{array}{l}\text { - Conocimiento olímpico } \\
\text { - Excelencia }\end{array}$ \\
\hline i) Comprender y expresarse en una o más lenguas extranjeras de manera apropiada. & $\begin{array}{l}\text { - Conocimiento olímpico } \\
\text { - Excelencia }\end{array}$ \\
\hline j) Conocer, valorar y respetar los aspectos básicos de la cultura y la historia propias y de los demás, así como el patrimonio artístico y cultural. & $\begin{array}{l}\text { - Amistad } \\
\text { - Respeto } \\
\text { - Conocimiento olímpico }\end{array}$ \\
\hline $\begin{array}{l}\text { k) Conocer y aceptar el funcionamiento del propio cuerpo y el de los otros, respetar las diferencias, afianzar los hábitos de cuidado y salud corporales e incorporar la } \\
\text { educación física y la práctica del deporte para favorecer el desarrollo personal y social. Conocer y valorar la dimensión humana de la sexualidad en toda su diversidad. } \\
\text { Valorar críticamente los hábitos sociales relacionados con la salud, el consumo, el cuidado de los seres vivos y el medio ambiente, contribuyendo a su conservación y } \\
\text { mejora. }\end{array}$ & $\begin{array}{l}\text { - Excelencia } \\
\text { - Respeto } \\
\text { - Conocimiento olímpico }\end{array}$ \\
\hline l) Apreciar la creación artística y comprender el lenguaje de las distintas manife & $\begin{array}{l}\text { - Amistad } \\
\text { - Respeto } \\
\text { - Conocimiento olímpico }\end{array}$ \\
\hline
\end{tabular}


evitando aislar nuestro proyecto de educación olímpica en una única asignatura.

La finalidad fundamental es difundir los valores olímpicos, haciendo partícipes a los alumnos y alumnas de su propio aprendizaje y protagonistas de las tareas de sensibilización y promoción de valores en nuestro centro educativo.

Enumeramos a continuación los objetivos específicos de Olimpízate:

1. Abordar cuatro dimensiones de aprendizaje (conocimiento olímpico, excelencia, amistad y respeto) desde una perspectiva interdisciplinar para propiciar un aprendizaje significativo.

2. Difundir los valores olímpicos, estimulando la imaginación y el aprendizaje de los estudiantes.

3. Participar en actividades de sensibilización y proyección de valores en nuestro centro educativo.

4. Adquirir una conciencia crítica sobre el Olimpismo y el tratamiento de la información en el mundo del deporte, especialmente en la prensa deportiva.

5. Utilizar actividades orientadas a la resolución de problemas, donde los alumnos y alumnas sean los protagonistas y artífices de su propio aprendizaje.

6. Utilizar el potencial de la competición deportiva para que los valores olímpicos puedan ser experimentados y vividos por los propios estudiantes.

\section{El proyecto educativo Olimpízate. Desarrollo}

Olimpízate se desarrolló a lo largo de 6 semanas entre los meses de marzo y abril y durante el curso escolar de 2015/ 2016. Las actividades propuestas desde cada materia pudieron ejecutarse dentro y fuera del centro educativo. En nuestro IES, cada profesor utilizó aulas específicas: sala de ordenadores, taller de tecnología, aula de dibujo, aula específica del grupo, pabellón y pista polideportiva. En cuanto a las ubicaciones fuera del centro, los alumnos se valieron de dos parques públicos, la biblioteca e instalaciones deportivas municipales. Las tareas desarrolladas fuera del horario lectivo fueron contempladas como actividades Tabla 3.

\begin{tabular}{|c|c|c|}
\hline \\
\hline $\begin{array}{l}\text { Histo } \\
\text { Hist }\end{array}$ & \multicolumn{2}{|c|}{$\begin{array}{l}\text { Actividades } \\
\text { A partir de la lectura de un artículo online (http://www.am14.net/jesse-owens-y- } \\
\text { luz-long-amistad-por-encima-del-color-de-la-piel//) sobre la amistad surgida en } \\
\text { los Juegos de Berlín } 1936 \text { entre Jesse Owens y Luz Long, los alumnos y alumnas } \\
\text { trabajan a través de Webquest los siguientes temas: } \\
\text { - Conflictos bélicos en el siglo XX. } \\
-\quad \text { El nazismo en Europa. }\end{array}$} \\
\hline $\begin{array}{l}\text { iología y } \\
\text { Geología }\end{array}$ & \multicolumn{2}{|r|}{$\begin{array}{l}\text { Sustancias dopantes en el deporte. Análisis de sus consecuencias a corto, medic } \\
\text { y largo plazo. } \\
\text { - Estudio de caso: Alex Schwazer, ganador del oro olímpico en Pekín } 2008 \text { de } \\
\text { los } 50 \text { kilómetros marcha, fue excluido por dopaje del equipo olímpico } \\
\text { italiano de Londres } 2012 \text {. } \\
\text { - ¿Por qué el dopaje atenta contra los principios éticos del deporte? }\end{array}$} \\
\hline & $\begin{array}{l}\text { Tres ficl } \\
\text { amistad } \\
\text { ordenad } \\
\text { para la } \\
\text { asociado } \\
\text { De esta } \\
\text { 1) "Def } \\
\text { cono } \\
\text { 2) "El t } \\
\text { un v } \\
\text { en el } \\
\text { 3) "La } \\
\text { refle } \\
\text { 4) "El } \\
\text { dich }\end{array}$ & $\begin{array}{l}\text { has (Figura 1) para reflexionar sobre los valores olímpicos (excelencia, } \\
\text { y respeto). Cada ficha, que se cumplimenta directamente desde el } \\
\text { or, dedica su contenido a cada uno de los valores y plantea actividades } \\
\text { búsqueda activa en internet y la reflexión sobre temas de actualidad } \\
\text { os al valor específico de estudio. } \\
\text { manera, las fichas contienen los siguientes apartados: } \\
\text { finiendo". El alumno deduce qué significa cada valor y aporta los } \\
\text { cimientos previos que tiene sobre el tema. } \\
\text { testimonio". El ejemplo de vida de un deportista, un artículo de prensa o } \\
\text { ídeo invitan al alumno a indagar en la pervivencia de un valor olímpico } \\
\text { ldeporte. } \\
\text { cita de...". Entrevistas de deportistas en medios de comunicación para } \\
\text { xionar sobre la dimensión pedagógica del Olimpismo. } \\
\text { día a día”. Invita a reflexionar sobre la manera en la que extrapolamos } \\
\text { os valores a nuestra vida cotidiana. }\end{array}$ \\
\hline \multicolumn{3}{|c|}{$\begin{array}{l}\text { Tabla } 4 . \\
\text { Materias y actividades de las sesiones del desafío. Los Reporteros del } \\
\text { Olimpízate. }\end{array}$} \\
\hline \multicolumn{3}{|c|}{$\begin{array}{l}\text { Planteamiento: } \\
-\quad \text { Artículo de prensa original sobre un evento deportivo de actualidad. } \\
-\quad \text { Aproximadamente } 150 \text { palabras (en castellano o en inglés). } \\
\text { - Incidir en los valores olímpicos y gestos ejemplificadores. }\end{array}$} \\
\hline \multicolumn{3}{|c|}{ Materia } \\
\hline $\begin{array}{c}\text { gua } \\
\text { lite }\end{array}$ & & $\begin{array}{l}\text { Redacción de un artículo de prensa, organizado en las } \\
\text { - } \text { Titular } \\
\text { - } \text { Autor } \\
\text { - Cuerpo del artículo (introducción, ideas prin } \\
\text { secundarias) } \\
\text { - Conclusión } \\
\text { - Fotografía }\end{array}$ \\
\hline & & a artículo de prensa oroanizado on las partes orientat \\
\hline
\end{tabular}

Tabla 5.

Materias y actividades de las sesiones del desafí.o Los Ojeadores del Proyecto Educativo

Olimpízate.

Crear un nuevo sistema de puntuación para la competición deportiva de ámbito escolar que implique a todos los agentes que intervienen: jugadores, público, entrenadores y árbitro. Actividades
Materia Matemáticas comportamiento y la actitud de jugadores, espectadores, entrenadores y árbitros en el campo de juego. El objetivo es concienciar a todos los Tecnologías de la integrantes de un evento deportivo acerca de su responsabilidad en la Terón dén y jóvenes.

\begin{tabular}{c} 
comunicación \\
cormación y ducación de niños y jovenes. \\
\hline
\end{tabular}

- An in Jara $\begin{array}{ll}\text { Educación física } & \begin{array}{l}\text { Android Juga Verd Play, desarrollado para la competición deportiva } \\ \text { escolar en la comarca del Baix Llobregat, Barcelona. }\end{array}\end{array}$

Tabla 6 .

Materias y actividades de las sesiones del desafío. Cortometraje del Proyecto Educativo Olimpizate.

Planpizate.

Grabación de un cortometraje acerca de cómo entienden los alumnos y alumnas los valores olímpicos y cómo pueden proyectarlos en el centro educativo.

Duración máxima: 3 minutos.

Todas las partes del cortometraje deben ser de elaboración propia: tomas, edición, música, vestuario, etc.

Grupos de 4 a 7 alumnos y alumnas.

Materia

Música - Ensayo y grabación de las piezas musicales. Utilización de flauta dulce y percusión.

Dos temas musicales por grupo.

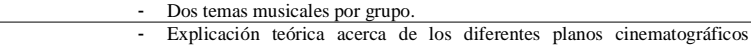

Educación (plano general, plano medio, plano americano, primer plano, primerísimo

pástica, visual plano y plano detalle), así como los criterios para su utilización en el cine.

y audiovisual - Grabación de tomas en los espacios del centro educativo y alrededores (siempre en horario escolar).
(siono

Desde esta materia se procede a la edición del cortometraje en base a las tomas de vídeo y la música grabadas. Para ello, se les propone la posibilidad cnologías de de hacerlo mediante diversos programas informáticos:

información - Pinnacle

y de la - Movie Make

comunicación - Sony Vegas

Programas de edición en Android: Video Director

Programas de edición en Apple: iMovi

Los cortometrajes son visualizados en las clases de esta materia y sirven de preámbulo para trabajar, mediante exposición de ideas y debate, sobre lo problemas más candentes que afectan a la convivencia humana y cómo los valores olímpicos, transmitidos a través del deporte, pueden favorecer para

Valores éticos encontrar soluciones. Así, se exponen temas como: Inmigración (crisis de los refugiados en Europa) Autoestima.

Superación personal.

Relaciones humanas. El deporte como vehículo para la paz y el entendimiento.

curriculares, estableciendo cada docente y desde cada asignatura cuáles, cuándo y de qué manera debían desarrollarse.

Para la consecución de los objetivos del proyecto, organizamos a los alumnos y alumnas en pequeños grupos y organizamos todas las actividades de enseñanza y aprendizaje en torno a tres retos globales y plenamente interdisciplinares. Nuestro propósito era motivar desde un principio a los estudiantes y la idea de plantearles tres desafíos desarrollados desde distintas asignaturas, nos pareció la fórmula más acertada para incentivar su compromiso con Olimpízate y para que la participación de cada una de las materias fuera imprescindible para la correcta consecución del reto. Los tres desafíos propuestos fueron: 1) Los reporteros, 2) Los ojeadores y 3) Cortometraje. En las siguientes tablas hemos detallado las actividades planteadas en cada uno de ellos, así como las materias involucradas.

Antes de informarles sobre el desarrollo de estos retos, durante la primera semana de intervención, llevamos a cabo varias sesiones de evaluación inicial (encuesta sobre la percepción de los valores en el deporte y la simbología olímpica), conocimiento olímpico e introducción a los valores (Tabla 3).

Tras una primera semana de actividades de carácter teórico, planteamos a los alumnos y alumnas los tres retos. Cada uno de ellos fue abordado desde diversas materias como podemos ver de forma resumida en la Tabla 4, Tabla 5 y Tabla 6.

\section{Resultados y decisiones de acción para la próxima puesta en práctica}

Al finalizar el proyecto, volvimos a facilitar a los alumnos y alumnas la encuesta de evaluación inicial sobre la percepción de los valores en el deporte y la simbología olímpica, con el ánimo de conocer los posibles cambios en las respuestas. Igualmente, planteamos a los profesores implicados en Olimpízate un cuestionario para observar su grado de 
satisfacción con esta iniciativa. Los resultados fueron positivos y pueden resumirse en estos datos:

- El 91\% de los docentes cree que el proyecto motivó en mayor medida a los alumnos y alumnas en sus materias.

- El 83\% de los docentes volvería a plantear un proyecto similar en el próximo curso escolar.

Esta experiencia nos ha permitido explorar y poner en práctica las enormes posibilidades que tiene un centro educativo para incentivar la educación olímpica en los alumnos y alumnas de secundaria. Durante las semanas en las que se desarrolló esta iniciativa, pudimos corroborar una serie de ventajas pedagógicas, como la mayor implicación de los alumnos y alumnas en asignaturas a las que hasta entonces dedicaba poco interés, un clima colaborativo favorecido por la organización en pequeños grupos, y finalmente, una especial disposición de los docentes para participar en este tipo de proyectos, que suponen un cambio de perspectiva en la manera de abordar los contenidos desde sus asignaturas. Estos aspectos positivos, desde el punto de vista educativo, también fueron reflejados en similares experiencias interdisciplinares recientes (Peixoto, 2014; Binder, 2010).

Sin embargo, también hemos encontrado una serie de dificultades en el desarrollo de Olimpízate: la primera de ellas es la necesaria implicación de los docentes. Una iniciativa de esta envergadura precisa de horas de trabajo para la preparación de materiales específicos, que no siempre están disponibles en el mercado (desde partituras de melodías en las clases de música, hasta la confección de fichas sobre los valores olímpicos, que recojan casos cercanos y actuales). No todos los profesores estuvieron igual de dispuestos para la elaboración de nuevos materiales y la consecuente alteración de su metodología de enseñanza, seguida durante numerosos cursos escolares. Aunque finalmente pudimos solventar estos escollos, gracias a la colaboración entre compañeros de trabajo y la facilidad de contar con un claustro de profesores pequeño, esto podría suponer un importante condicionante en centros más grandes a la hora de crearun proyecto verdaderamente interdisciplinar. La segunda limitación que encontramos es la propia organización de las leyes educativas actuales, que a nuestro parecer, se posicionan en la tendencia de individualizar la estructura curricular de cada una de las asignaturas, restringiendo la facilidad de crear este tipo de experiencias colaborativas, mucho más acordes con la realidad social y laboral que los estudiantes van a encontrar al finalizar la etapa de la ESO.

Con todo, creemos que tanto Olimpízate, como otros proyectos elaborados en la misma línea, pueden ser muy positivos para un centro educativo, siempre y cuando contemos con docentes innovadores y concienciados con las ventajas de una educación olímpica en el entorno escolar. Podemos señalar de forma resumida los pasos a seguir para llevar a cabo un proyecto similar:

1. Definir los contenidos olímpicos. En nuestro caso, Olimpízate ha recogido los valores olímpicos promovidos por el COI (excelencia, amistad y respeto) al que hemos sumado un cuarto contenido titulado «conocimiento olímpico». A todos ellos los hemos denominado dimensiones pedagógicas. Esta clasificación es importante para establecer una correlación lógica con los elementos curriculares y para centrar la actuación de las actividades didácticas.

2. Concretar el grado de interdisciplinariedad. Para conseguir aprendizajes más globales y significativos, este proyecto ha apostado por la plena implicación de las materias cursadas en $4^{\circ} \mathrm{de}$ ESO. Sin embargo, esta experiencia podría presentar más dificultades en centros educativos grandes o donde el equipo docente esté poco habituado a proyectos interdisciplinares.

3. Crear materiales didácticos. Internet y los modelos educativos de temática olímpica desarrollados en el mundo y de acceso libre son una inagotable fuente de inspiración para confeccionar materiales acordes al contexto educativo donde se desenvuelven los estudiantes. En la Figura 2 compartimos en código QR algunos de los materiales y herramientas elaborados para Olimpízate.

4. Desarrollar el proyecto. La puesta en práctica de las actividades y su evolución a lo largo de las sesiones proporcionará información acerca de los aspectos a mejorar y aquellos que han servido especial- mente para lograr nuestros objetivos.

\section{Conclusiones}

Como hemos podido comprobar, Olimpízate no es solo un proyecto interdisciplinar en el que se lleva el Olimpismo a las aulas de forma superficial, a base de conceptos, clases magistrales de atletas deélite o la elaboración de pósters con mensajes idilicos acerca de las bondades del deporte y de su gran utilidad a la sociedad. Tampoco se limita exclusivamente a las muy difundidas y mal denominadas «Olimpiadas Escolares», entendidas como jornadas o semanas específicas en las que los estudiantes participan en una serie de competiciones deportivas, e incluso en las que se escenifican los rituales de una ceremonia de apertura en unos Juegos Olímpicos (desfile de los atletas o el encendido de un pebetero).

La gran aportación de esta iniciativa es la metodología seguida en el desarrollo de las actividades propuestas. Ya advertíamos en los objetivos específicos que nuestra finalidad era hacer a los estudiantes partícipes de su propio aprendizaje y que los valores olímpicos no fueran expuestos de manera únicamente teorizada, sino que pudieran ser vividos y filtrados a través de su propia experiencia, para que finalmente, nuestros alumnos y alumnas fueran los artífices de la difusión de los mismos en el centro educativo. El deporte, como no podía ser de otra manera, ha sido la correa transmisora que nos ha permitido adentrarnos en el mundo de los valores; pero además nos hemos valido de la complementariedad que las competiciones deportivas pueden tener con el arte, la historia, la ciencia y la tecnología, y así hacer de este proyecto un modelo más atractivo y dinámico.

Olimpízate se ha llevado a cabo de manera experimental en un centro concreto, con un número muy reducido de profesores y alumnos. En nuestro ánimo está llevar este proyecto interdisciplinar a otros centros cercanos de la Sierra del Segura albaceteña e incluso a la vecina comarca del Noroeste, en la Región de Murcia. De esta forma, habida cuenta de los resultados obtenidos en los cuestionarios entregados a los alumnos, podríamos conocer los posibles cambios en la percepción respecto a los valores olímpicos, contando, eso sí, con una población más representativa que avalara las conclusiones.

\section{Referencias}

Binder, D. (2007). Teaching values. An olympic education toolkit. Lausanne: International Olympic Committee.

Binder, D. (2010). Olimpismo en las escuelas: la Educación Olímpica como

centro de la educación en valores. Lecciones universitarias olímpicas. Barcelona: Centre d'Estudis Olímpics (UAB). Cátedra Internacional de Olimpismo. Recuperado de http://olympicstudies.uab.es/ 2010/docs/binder_spa.pdf

Calle, M.T., \& Martínez, M.E. (2015). Los valores del Movimiento Olímpico en la educación física: su contenido y presencia en la Educación Secundaria Obligatoria en España. Materiales para la Historia del Deporte, Suplemento Especial (2), 357-363.

Chatziefstathiou, D. y Henry, I. (2012). Discurses of Olympism: From the Sorbone 1894 to London 2012. Londres: Palgrave Macmillan.

Coubertin, P. (2012). La educación inglesa. Citius, Altius, Fortius: $\mathrm{Hu}$ manismo, Sociedad y Deporte: Investigaciones y ensayos, 5(1), 133-151.

Culpan, I., \& Wigmore, S. (2010). The Delivery of Olympism Education within a Physical Education Context Drawing on Critical Pedagogy. International Journal of Sport and Health Science, (8), 67-76.

Dacosta, L. (2002). Olympic Studies: Current Intellectual Crossroads. Río de Janeiro: Gama Filho.

Defroand, J. (2012). London 2012: olympic 'legacy’, olympic education and the development of social capital in physical education and school sport: a case study (Tesis doctoral). University of Birmingham, Birmingham.

Hernández, Y. (2014). Deporte escolar y educación en valores: 
fundamentación desde un enfoque sociocultural y pedagógico. Materiales para la Historia del Deporte, (12), 111-134.

Herrero, D. (2015). La Educación Olímpica: propuesta interdisciplinar en la Enseñanza Secundaria Obligatoria. Materiales para la Historia del Deporte. Suplemento Especial, (2), 241-256.

IOC, Olympic Charter (Updated, Augusto 2015), International Olympic Committee. Lausanne. Recuperado de https://www.olympic.org/ olympic-studies-centre/collections/official-publications/olympiccharters

Lenk, H. (1972). Werte, Ziele, Wirklichkeit der modernen Olympischen Spiele. Schorndorf: Hofmann.

Lioumpi, P., Georgiadis, K., \& Mountakis, C. (2014). La aplicación de un programa de intervención en educación física para alumnos griegos de sexto de educación primaria: difusión de los valores y actitudes del ideario del Movimiento Olímpico. Citius, Altius, Fortius, 7(1) 67-97.

Lucas, J. (1978). The modern Olympic Games. New Jersey: Barnes.

Maass, S. (2007). The Olympic Values, Olympic Review, (63), 28-33.

Maillard, C., \& Monnin, É. (2014). Une éducation à l’olympisme estelle possible? Éducation et socialisation. Les Cahiers du CERFEE, (36)

Makris, A., \& Georgiadis, K. (2013). Los Juegos Olímpicos de la Juventud: una nueva institución del Movimiento Olímpico para la difusión de los valores educativos del Olimpismo, Citius, Altius, Fortius, 6(2), 76-95.

Martínez, M. (2011). Educación, valores y democracia. Revista de Educación, Número Extraordinario 2011, 15-19.

Martínez, M.J., Pena-Pérez, X., \& Mateos, C. (2016). Percepción en adolescentes de los valores olímpicos asociados a la práctica del fútbol frente a otros deportes. Retos. Nuevas Tendencias En Educación Física, Deporte y Recreación, (30), 226-232.

Meroño, L., Bada, J., Sánchez-Pato,A., Calderón,A., \& Figuer-Montero, C. (2016). Más rápido, más alto, más fuerte: percepción del alumnado de Educación Secundaria sobre sus actitudes y comportamientos a través del Aprendizaje Basado en Problemas. Rivista Italiana di Pedagogia dello Sport, 1, 13-28.

Monjas, R., Ponce, A., \& Gea, J.M. (2015). La transmisión de valores a través del deporte. Deporte escolar y deporte federado: relaciones, puentes y posibles transferencias. Retos. Nuevas Tendencias En Educación Física, Deportey Recreación, (28), 276-284.

Naul, R. (2008). Olympic Education. Londres: Meyer \& Meyer.

Naul, R. (Ed.)., Binder, D. (Ed.)., Rychtecky, A. (Ed.)., \& Culpan, I. (Ed.). (2017). Olympic Education: An international review. Abingdon, Reino Unido: Routledge.

Nikolaus, I. (2013). La educación olímpica en el mundo, Citius, Altius, Fortius, 6(2), 58-75.

Ouergli, F. (2014). La promotion des valeurs olympiques au sein des communaut_es virtuelles: le cas de Facebook (Tesis doctoral). Universidad de Niza Sophia Antipolis, Niza.

Peixoto, L. (2014). Proyecto interdisciplinar, Escuela: Comunidad Olímpica, Retos. Nuevas Tendencias en Educación Física, Deporte y Recreación, (25), 140-143.

Prat, M., Soler, S., Ventura, C., y Tirado, M. A. (2011). El deporte como instrumento para el desarrollo de la capacidad crítica de los adolescentes. En J. Durán (Ed.), Valores en movimiento: la actividad física y el deporte como medio de educación en valores, (pp. 127-160). Madrid: Consejo Superior de Deportes.

Rocha, T.C., Martínez, M.J., Mateos, C., \& Pena-Pérez, X. (2016). El Olimpismo en la formación de entrenadores deportivos en Portugal. Retos. Nuevas Tendencias En Educación Física, Deporte y Recreación, (30), 233-236.

Shcherbashyn, I. S. (2014). Olympic education as a method of humanistic elevation of students. Pedagogics Psychology, 7(2), 59-70.

Solar, L. (2003). Pierre de Coubertin. La dimensión pedagógica. Madrid: Gymnos.

Tavares, O. (2006). Los valores olímpicos en el siglo XXI: entre la continuidad y el cambio. Lecciones universitarias olímpicas. Barcelona: Bellaterra. Recuperado de http://olympicstudies.uab.es/ lectures/web/pdf/tavares.pdf.

Thorn, S. (2010). OlympismEducation: Teaching and learning Olympism in a New Zealand secondary physical education programme(Tesis doctoral). Universidad de Canterbury, Canterbury.

Weiss, O. (2012). Identity and (democratic) values in sport, The Official Journal of the International Olympic Academy, (3), 26-29.

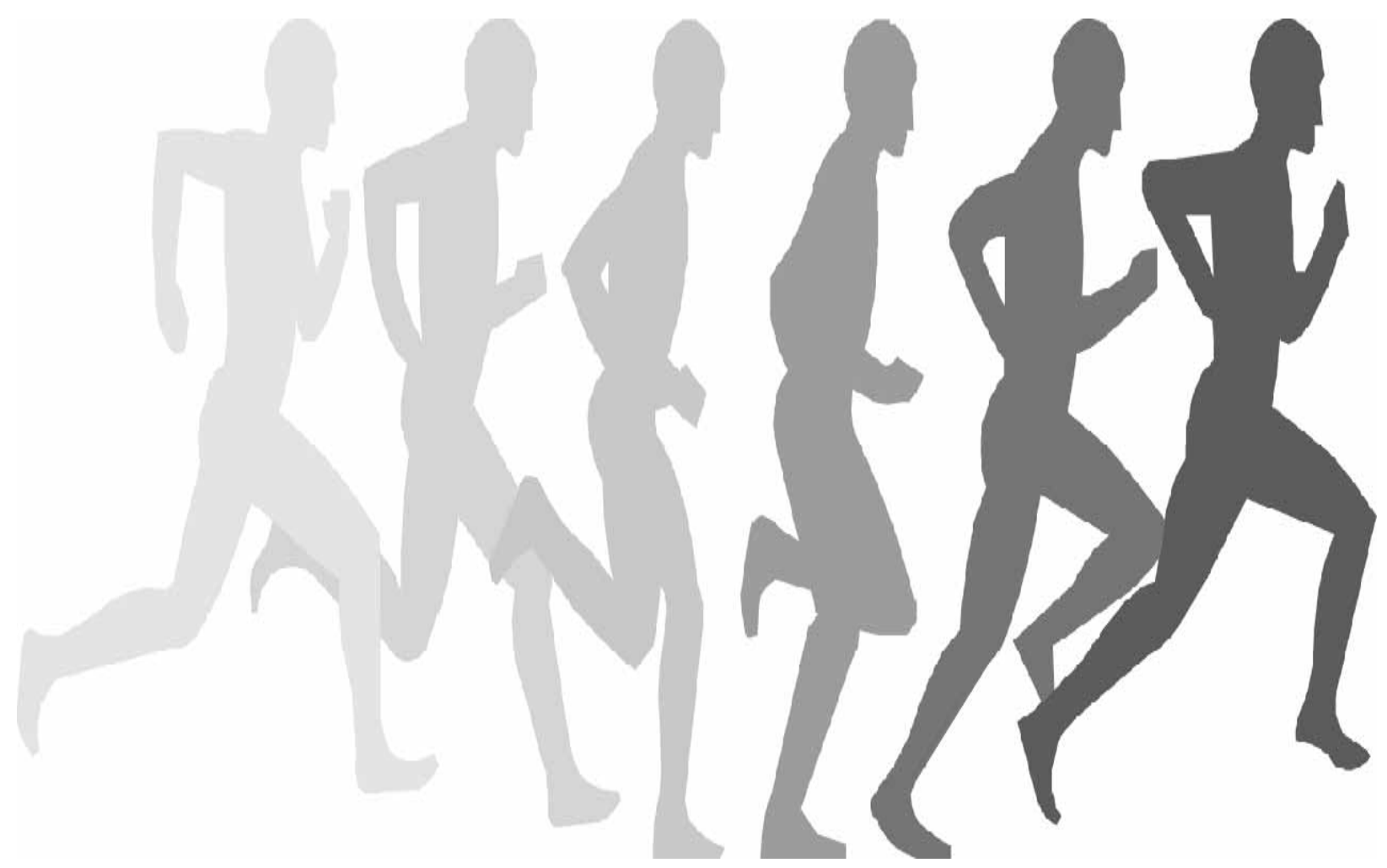

